\title{
METHOD OF PARTITIONING THE TERRITORY FOR LOGISTIC SOLUTIONS SUPPORT BASED ON GENETIC CLUSTERING
}

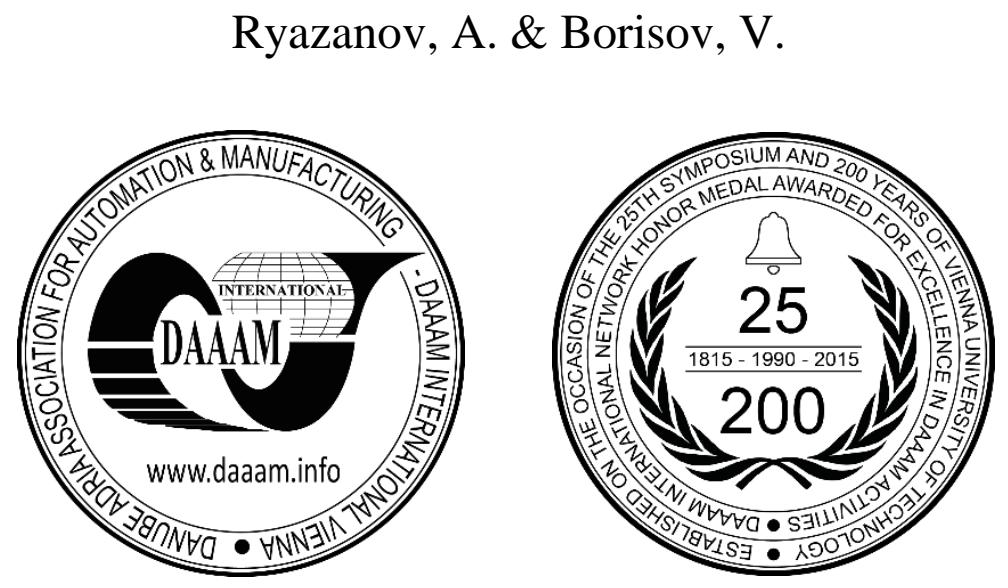

This Publication has to be referred as: Ryazanov, A[rtem] \& Borisov, V[adim] (2018). Method of Partitioning the Territory to Logistic Solutions Support Based on Genetic Clustering, Proceedings of the 29th DAAAM International Symposium, pp.0961-0965, B. Katalinic (Ed.), Published by DAAAM International, ISBN 978-3-902734-20-4, ISSN 1726-9679, Vienna, Austria

DOI: $10.2507 / 29$ th.daaam.proceedings. 138

\begin{abstract}
The article proposes an original method of zonal partition of the territory for logistic solutions support based on genetic clusterization. The method includes: firstly, the search of territorial zones (regions) with high intensity of logistics orders and the centers of these areas; secondly, determination of the optimal number of areas for the territory; thirdly, the partition of the territory into zones using the developed algorithm of genetic clusterization. The originality of the proposed method lies in the fact that territorial objects (houses) are used as individuals of the genetic clusterization algorithm, and genetic operations are represented by the movement of individuals on the boundaries of adjacent zones. A comparative analysis of the proposed method with known methods of zonal partition of territories using Voronoi diagrams is performed. The results of this analysis showed that the proposed method makes it possible to improve the quality and efficiency of the logistics decisions made by taking into account uncertainties arising from additional characteristics of the territory (natural barriers, administrative divisions, etc.).
\end{abstract}

Keywords: clustering genetic algorithm; distribution of logistic orders; zonal partition of the territory; Voronoi algorithm.

\section{Introduction}

The qualitative and operative solution of logistics tasks, with the use of navigation aids (for example, the GPS system) is now becoming more important and relevant. Most of these tasks require an optimal partition of the territories into logistics service zones based on the intensity of the logistics orders and the capabilities of the logistics service to ensure these orders. One of the most popular approaches for zonal partition is the use of Voronoi diagrams [1]. However, despite the promptness of the solution of this problem, the use of Voronoi diagrams does not allow us to take into account a number of uncertainties due to additional characteristics of the territory. As a result, it leads to a decrease in the quality of the accepted logistics solutions [2], [3]. The article proposes a new method of zonal partition of the territory for logistics solutions support, based on the genetic clusterization of territorial objects. The method provides an optimal partition of the territory into the zones, taking into account the intensity of logistic orders, additional characteristics of the territory (natural barriers, administrative divisions, etc.), as well as the possibilities of logistics facilities. 


\section{Method of zonal partition of the territory based on genetic clusterization}

Most of the known methods of zonal partition of territories are based on Voronoi diagrams. The Voronoi diagram on a plane represents a partition of the plane in which each region of this partition forms a set of points closer to one of the elements of this set than to any other element of the set. The regions of the partition are convex polygons. For the partition of a territory using the Voronoi diagram, it is necessary to limit the area of the partition and find the coordinates of the points around which the regions (zones) will be built. An example of partition of a territory using the Voronoi diagram is shown in Figure 1. The main advantage of these methods is their efficiency, due to the estimation of computational complexity - from $O(n \times \log (n))$ to $O(n)$.

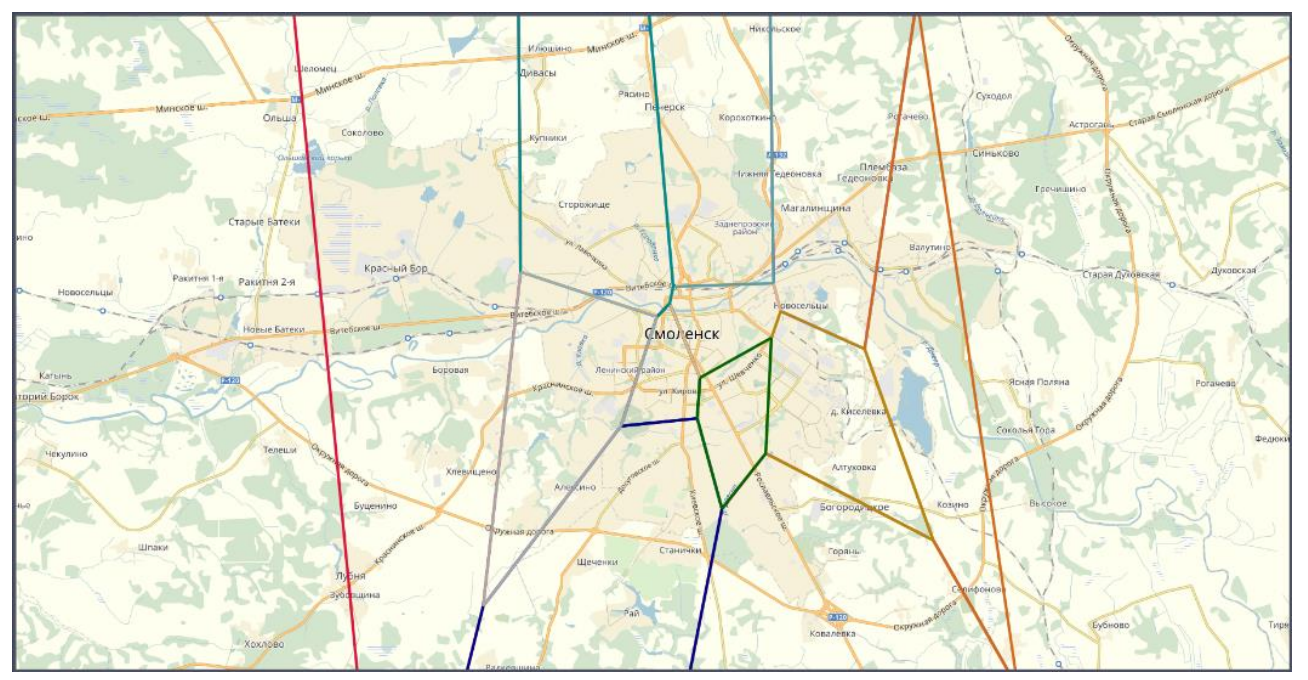

Fig. 1. An example of partitioning of the territory using the Voronoi diagram

However, for the zonal partition of the territory using the Voronoi diagrams, only the coordinates of the bounding region and the coordinates of the points around which the regions are formed are taken into account [4], [5]. In the paper [6] the procedure of the zonal partition of the territory is considered. The zonal partition can be represented as follows:

$$
R=\left\{R_{i}\right\}, i=1, \ldots, I, \quad \forall R_{i}=\left\{r_{n_{i}}\right\}, n_{i}=1, \ldots, N_{i},
$$

where $R$ - a set of zonal partitions of the territory, $R_{i}$ - zonal partition of the territory for $i$-th time (usually, $i$-th hour) of the day, $i=1, \ldots, I$ (for the problem being solved $I=24$ ), $r_{n_{i}}$ - a separate zone of the zonal partition $R_{i}, n_{i}=1, \ldots, N_{i}, I-$ a number of possible variants of zonal partitions. For each zone $r_{n_{i}}, n_{i}=1, \ldots, N_{i}$ a zonal partition $R_{i}$ refers to a subset $H_{n_{i}}$ of territorial objects (houses) $h_{k_{j}}$ :

$H_{n_{i}}^{\left(R_{i}\right)}=\left\{h_{k_{i}}^{\left(n_{i}\right)}\right\}, k_{i}=1, \ldots, K_{i}$

where $k_{i}$ - a number of territorial objects in the zone $r_{n_{i}}$ of the zonal partition $R_{i}$. The aggregate of all the territorial objects related to the zonal partition $R_{i}$, is marked as:

$$
H_{R_{i}}=\left\{H_{n_{i}}^{\left(R_{i}\right)}\right\}, n_{i}=1, \ldots, N_{i}
$$

The zonal partition is carried out on the basis of known coordinates of territorial objects and the number of orders received for a certain period. For this purpose it is preliminary supposed to determine the number of the orders received from all the territorial objects, to identify the areas with a high intensity of orders and the assignment of these objects to the corresponding zones. A separate zone includes many objects located in the territorial proximity to the center of the thickening area of orders. Technologies of evolutionary modeling and genetic algorithms are provide: rapid convergence at decision of optimizing problems; various types of parameters can be taken into account; possibility of use of expert, empirical, rough and uncertain data; possibility of nonlinearity representation and modelling; possibility of fast results updating; good adaptation properties [7], [8], [9]. The proposed method of zonal partition of the territory is based on the use of genetic clusterization. For the operation of this method, the objective function of the estimation of the fitness of individuals in the population is given in the first place. The criterion for the objective function is the reduction of the time of the arrival of the vehicle to the place of the logistics order. 
The zonal partition of territories based on the genetic clusterization for the $i$-th time of day is considered here:

Step 1. Specification of objective function (fitness) for individuals of a population.

Step 2. Creation of the initial population (using the $k$-means algorithm, the given number of clusters-zones is formed). Territorial objects belonging to the zones are recorded in the genotype of the individual appropriate to this zone.

Step 3. Crossing (it is represented by the exchange of territorial objects located on the border of adjacent zones, which leads to a "smooth" change in the boundaries of the zones).

Step 4. Mutation (an arbitrary movement of one or more territorial objects, located on the border, from one adjacent zone to another).

Step 5. Formation of a new generation (a selection of the zones with the best values of the objective function).

Step 6. If the criterion for stopping the algorithm is not satisfied, then return to the step 3.

The stopping conditions are:

- the maximum average value of fitness functions of all the individuals of the population;

- the achieving of the difference between the values of the objective functions of adjacent zones is less than the specified value.

According to the results of the zonal partition method, the zones, where the difference between the values of the objective functions of adjacent zones is the smallest, are formed. Thus, the distinctive features of the proposed method of zonal partition of territories based on genetic clusterization are the following:

- the flexibility of changing the shape of the zones and the values of the objective function due to the crossing represented by the "exchange of territorial objects" between the zones;

- the reduction of computational complexity of calculations in comparison with the Voronoi diagrams;

- the increase in the dimension of the problem does not significantly affect the complexity on the whole and the complexity of the solution of the problem, in contrast to other methods of territorial partition, in which the complexity and labor intensity increase exponentially from the number of parameters;

- the combination of advantages of clusterization algorithms and genetic algorithms.

The use of the proposed method of genetic clusterization for solving the zonal partition problem does not guarantee the detection of an optimum in polynomial time. However, this method allows to choose a "sufficiently good" solution in less time than other known deterministic or heuristic algorithms of search optimization. Since the partition of the territory is performed taking into account the coordinates of known territorial objects, the distribution does not cover the whole territory of the partition. In order to get an order received from an unknown territorial object that does not belong to any zone of partition, the following algorithm is used:

Step 1. Finding the coordinates of the object using the geocoder of google maps.

Step 2. The distance from the territorial object to the centers of the edges and vertices of all zones is determined with the help of the coordinates found.

Step 3. The order from this object is distributed to the zone, in which the distance to the top or to the center of one of the edges turned out to be smaller, and the object itself is added to this zone.

As a result of the method, you can get a partition of the entire territory and the assignment of territorial objects into one of the zones, an example is shown in Figure 2.

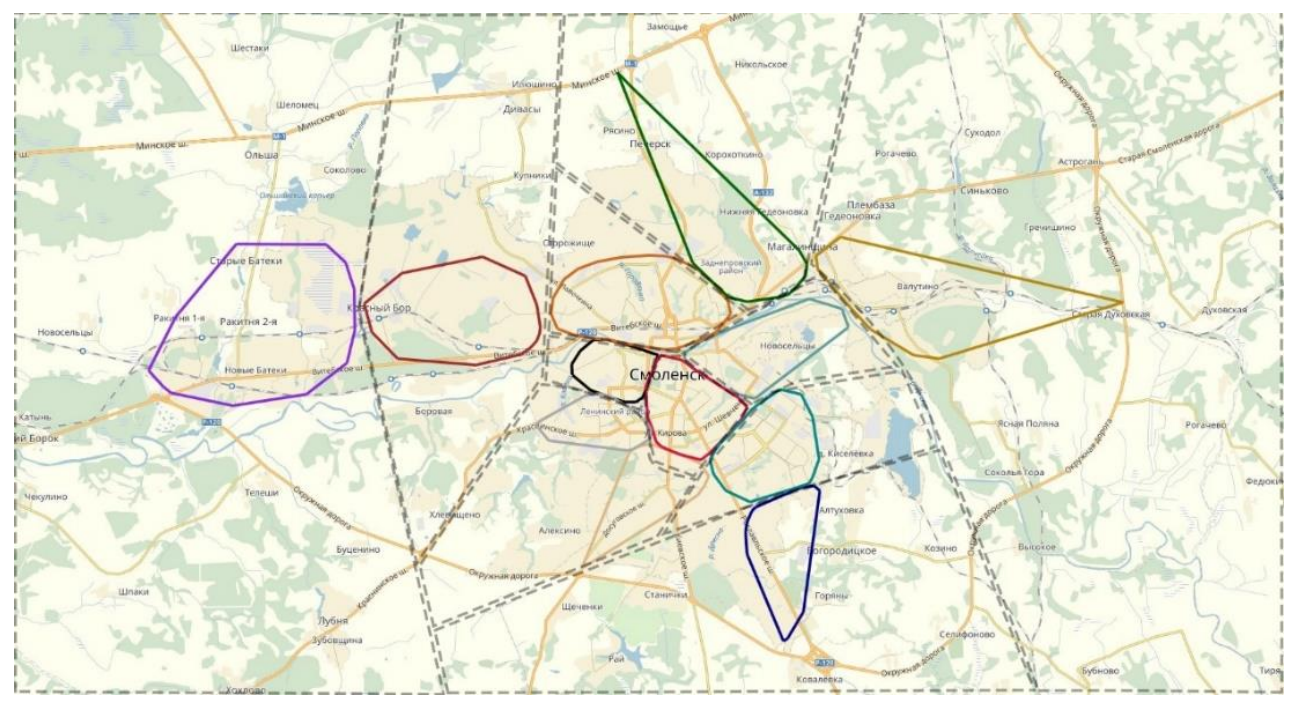

Fig. 2. An example of partitioning the territory by the genetic clustering algorithm, taking into account the distribution of the logistics orders 


\section{Analysis of the results of partitioning the territory into zones based on genetic clusterization}

For partition of the territory into zones, the key characteristics are:

- the size of the zone, which is important for how far the logistics facility can from the place of receipt of the order;

- the crossing of the zone by natural barriers (rivers, mountains), as well as the peculiarities of administrative division, which significantly affect the time of the fulfillment of the logistics order;

- the influence of the number of orders from the territorial objects in the respective zones on the "attractiveness" of these zones for logistics facilities;

Taking into account these characteristics, we will estimate the partition algorithms.

As a key criterion for the assessing quality, the maximization of orders in all zones for each hour is considered. The evaluation of the quality of zonal partition will be based on the standard deviation of the number of orders for all the zones of the partition for each hour of the day:

$Q=\sqrt{\frac{1}{n_{i}} \sum_{n_{i}=1}^{N_{i}}\left(k_{n_{i}}-k_{\text {avg }}^{\left(R_{i}\right)}\right)^{2}}$,

where $k_{n_{i}}$ - the number of orders for $n_{i}$-th zone of zonal partition of the territory $R_{i}, k_{\text {avg }}^{\left(R_{i}\right)}$ - the average number of orders for all zones of zonal partition $R_{i}$. The results of the comparative analysis of the zonal partition of the territory using the proposed method and the method based on Voronoi diagrams are presented in Table 1.

\begin{tabular}{|c|c|c||c|c|c|}
\hline \multirow{2}{*}{$\begin{array}{c}\text { Hour } \\
\text { of the day }\end{array}$} & $\begin{array}{c}\text { The standard deviation of the number } \\
\text { of orders for a zonal partition }\end{array}$ & \multirow{2}{*}{$\begin{array}{c}\text { Hour } \\
\text { of the day }\end{array}$} & $\begin{array}{c}\text { The standard deviation of the number } \\
\text { of orders for a zonal partition }\end{array}$ \\
\cline { 2 - 3 } & $\begin{array}{c}\text { using the Voronoi } \\
\text { diagrams }\end{array}$ & $\begin{array}{c}\text { using the proposed } \\
\text { method }\end{array}$ & & $\begin{array}{c}\text { using the Voronoi } \\
\text { diagrams }\end{array}$ & $\begin{array}{c}\text { using the proposed } \\
\text { method }\end{array}$ \\
\hline 1 & 4.431 & 3.955 & 13 & 6.902 & 7.181 \\
\hline 2 & 4.605 & 3.874 & 14 & 5.659 & 5.792 \\
\hline 3 & 2.433 & 2.825 & 15 & 5.347 & 5.077 \\
\hline 4 & 1.976 & 2.06 & 16 & 6.821 & 4.977 \\
\hline 5 & 1.71 & 1.801 & 17 & 10.593 & 10.36 \\
\hline 6 & 1.737 & 1.646 & 18 & 15.798 & 12.865 \\
\hline 7 & 3.603 & 3.223 & 19 & 18.215 & 15.212 \\
\hline 8 & 9.182 & 8.154 & 20 & 14.238 & 12.747 \\
\hline 9 & 12.257 & 10.613 & 21 & 14.428 & 13.295 \\
\hline 10 & 6.741 & 6.399 & 22 & 11.431 & 10.348 \\
\hline 11 & 7.026 & 6.547 & 23 & 12.413 & 11.51 \\
\hline 12 & 6.268 & 6.341 & 24 & 12.735 & 11.159 \\
\hline
\end{tabular}

Table 1. Results

The method of zonal partition of the territory based on genetic clusterization allows to increase the quality of the accepted logistics solutions by, on average, $9.46 \%$ compared to the method based on Voronoi diagrams.

\section{Conclusion}

The article proposes a method of zonal partition of the territory for logistic solutions support based on genetic clusterization. The method includes: firstly, the search of territorial zones (regions) with a high intensity of logistics orders and centers of these areas; secondly, the determination of the optimal number of areas for the territory; thirdly, the partition of the territory into zones using the developed algorithm of genetic clusterization. The originality of the proposed method lies in the fact that territorial objects (houses) are used as individuals of the genetic clusterization algorithm, and genetic operations are represented by the movement of individuals on the boundaries of adjacent zones. The original features of the proposed method of zonal partition of territories based on genetic clusterization are the following:

- the flexibility of changing the shape of the zones and the values of the objective function due to the crossing represented by the "exchange of territorial objects" between the zones;

- the reduction of computational complexity of calculations in comparison with the Voronoi diagrams;

- the increase in the dimension of the problem does not significantly affect the complexity on the whole and the complexity of the solution of the problem, in contrast to other methods of territorial partition, in which the complexity and labor intensity increase exponentially from the number of parameters;

- the combination of advantages of clusterization algorithms and genetic algorithms. 
A comparative analysis of the proposed method with known methods of zonal partition of territories using the Voronoi diagrams is fulfilled. The method of zonal partition of the territory based on genetic clusterization allows to increase the quality of the accepted logistics solutions by, on average, $9.46 \%$ compared to the method based on the Voronoi diagrams. The effect is achieved due to the consideration of uncertainties arising from additional characteristics of the territory (natural barriers, administrative divisions, etc.). The field of the application of this method is the distribution of logistics orders by logistics means for the operational transport services, for example, for taxi services.

In the future, the research results are planned to be used in transport services for the distribution of logistics orders taking into account the fuzziness. The use of the proposed method will increase the efficiency of solving the problem of logistics orders distribution, as well as reduce the computational load in the distribution of logistics orders due to a qualitative breakdown into service areas.

\section{Acknowledgements}

The work is conducted under the support of the Russian Science Foundation (project 16-19-10568).

\section{References}

[1] Aurenhammer, F., Klein, R., Lee, D.-T. (2013) Voronoi Diagrams and Delaunay Triangulations, World Scientific Publishing Co., Inc. River Edge, NJ, USA, ISBN: 9814447633.

[2] Wu J. (2012) Advances in K-means Clustering: A Data Mining Thinking (Springer Theses: Recognizing Outstanding Ph.D. Research), Springer-Verlag Berlin Heidelberg.

[3] Sonkin, D. M. (2009) Adaptive algorithm for distributing orders for servicing taxi cars, Izvestia Tomsk Polytechnic University, Vol. 315, No. 5, ISSN: 2500-1019, pp. 65-69 (in Russian).

[4] Samet, H. (1993) Applications of spatial data structures: computer graphics, image processing, and GIS, AddisonWesley, ISBN: 020150300X, 512 p.

[5] Sack, J. R., Urrutia, J. (1999) Handbook of computational geometry, North Holland, ISBN: 0444825371, 1088 p.

[6] Ryazanov, A. V.; Borisov, V. V. (2015) Construction of convex hulls for zonal partition of territories, In Proc. of XII-th Int. Scientific and Technical Conference of Students and Graduate Students, "Information Technologies, Power Engineering and Economics". Vol. 1, 2015, Smolensk: Universum, ISBN: 978-5-91412-257-4, pp. 289293. (in Russian).

[7] Sivanandam, S.N., Deepa, S. N. (2008) Introduction to Genetic Algorithms (PSG College of Technology), Springer-Verlag Gmbh, ISBN: 97836420922443642092241.442 p.

[8] Janeš, G., Car, Z. (2015) Usage of Partial Genome Fitness Evaluation Mechanism to Get Faster Results in Genetic Algorithms, Proceedings of the 25th DAAAM International Symposium, Elsevier Ltd, pp.1634-1639.

[9] Gregor, M., Krajčovič, M., Hnát, J., Hančinsky, V. (2016) Genetic Algorithms in the Design and Planning of Production System, Proceedings of the 26th DAAAM International Symposium, DAAAM International, ISBN 978-3-902734-07-5, ISSN 1726-9679, pp.0494-0500. 